\title{
INTEGRABILITY OF DOUBLY-PERIODIC RICCATI EQUATION
}

\author{
MA LING and GUAN KE-YING \\ Department of Applied Mathematics \\ Beijing University of Aeronautics and Astronautics \\ Beijing 100083, P.R. CHINA
}

(Received January 4, 1996 and in revised form June 6, 1997)

\begin{abstract}
By the structure of solvable subgroup of $S L(2, \mathbb{C})$ (see [1]), the integrability and properties of solutions of a Riccati equation with an elliptic function coefficient, which is related to a Fuchsian equation on the torus $T^{2}$, is studied.
\end{abstract}

KEY WORDS AND PHRASES: Integrability, doubly-periodic Riccati equation, monodromy group, solvable subgroup of $S L(2, \mathbb{C})$, invariant.

\section{AMS SUBJECT CLASSIFICATION CODES:}

\section{INTRODUCTION}

The research on the integrability theory of differential equations is obviously important and interesting both in theory and in application. Since the pioneering work of Abel and Galois for algebraic equations and the work of Liouville for a particular Riccati equation (see [2]), the theory of integrability of differential equations has been developed very fast in several different ways, such as the Lie group theory (see [3]), differential algebra (see [4]) and the monodromy group theory created by Poincare and Klein (see [5,6]), the theory of invariants of the transformation group (see [7]), etc. However, the investigation into the differential equations is unlike that of algebraic equations for which there is a unified and well developed Galois theory Because of the complexity, these different integrability theories of differential equations have not been unified, and for each theory there exist some fundamental difficulties (see $[1,6,8]$ ). Even in differential algebra, the concept of integrability for a Fuchsian system has not been developed quite clearly (see $[1,6]$ )

In this paper, we develop the results of [1] and [9] to give a clear interpretation to the integrability of a doubly periodic Riccati equation by the solvability of its monodromy group.

Consider the Riccati equation

$$
z^{\prime}=z^{2}-\lambda \wp(t),
$$

where the parameter $\lambda \in \mathbb{R}^{+}, t \in \mathbb{C}, z(t) \in \mathbb{C}$, and $\wp(t)$ is the Weierstrass elliptic function given by the differential equation

$$
\wp^{\prime 2}=4 \wp\left[\wp^{2}-1\right], \wp(0)=0 .
$$

It is known that $\wp(t)$ has the following properties: (i) $\wp(t)$ is doubly periodic with periods $\omega_{1}=2 \alpha \in \mathbb{R}$ and $\omega_{2}=i \omega_{1}$; (ii) $\wp(i t)=-\wp(t), \forall t \in \mathbb{C}$, (iii) for any $t \in \mathbb{R}, \wp(t) \in \mathbb{R}, \wp(-t)=\wp(t)$, and for $t \in[-\alpha, 0], \wp(t)$ increases from -1 to 0 ; (iv) the point $P_{1}=-\alpha-i \alpha$ is a pole of order two, and there are no other singularities except $P_{1}$ in the period parallelogram (see [10])

Because of the double-periodicity of $\wp(t)$, the equation (1) can be treated as a Riccati equation on the torus $T^{2}$ which is formed from the period parallelogram of $\wp(t)$ 
As we know (see $[1,9]$ ), by the transformation $z=-u^{\prime} / u$, the solution of the equation (1) is related to the following Fuchsian equation on the torus $T^{2}$

$$
u^{\prime \prime}-\lambda \wp(t) u=0
$$

Suppose that $\left(u_{1}(t), u_{2}(t)\right)$ is a fundamental solution system of equation (3), then there exist two matrices $A_{1}=\left(\begin{array}{ll}a_{1} & b_{1} \\ c_{1} & d_{1}\end{array}\right)$ and $A_{2}=\left(\begin{array}{ll}a_{2} & b_{2} \\ c_{2} & d_{2}\end{array}\right)$ which belong to the special linear group $S L(2, \mathbb{C})$, such that

$$
\begin{gathered}
\left(u_{1}(t+2 \alpha), u_{2}(t+2 \alpha)\right)=\left(u_{1}(t), u_{2}(t)\right) A_{1}, \\
\left(u_{1}(t+2 i \alpha), u_{2}(t+2 i \alpha)\right)=\left(u_{1}(t), u_{2}(t)\right) A_{2} .
\end{gathered}
$$

The group $G$ generated by $A_{1}$ and $A_{2}$, i.e. $G=\left\langle A_{1}, A_{2}\right\rangle$, is just the monodromy group of equation (3) (see $[1,9])$.

By means of this fundamental solution system $\left(u_{1}(t), u_{2}(t)\right)$ of equation (3), each solution $z(t)$ of the Riccati equation (1) can be expressed as

$$
z(t)=-\frac{\delta u_{1}^{\prime}(t)+u_{2}^{\prime}(t)}{\delta u_{1}(t)+u_{2}(t)}
$$

where $\delta \in \widehat{\mathbb{C}}=\mathbb{C} \cup\{\infty\}$. Moreover, we have

$$
\begin{aligned}
& z(t+2 \alpha)=-\frac{f_{1}(\delta) u_{1}^{\prime}(t)+u_{2}^{\prime}(t)}{f_{1}(\delta) u_{1}(t)+u_{2}(t)} \\
& z(t+2 i \alpha)=-\frac{f_{2}(\delta) u_{1}^{\prime}(t)+u_{2}^{\prime}(t)}{f_{2}(\delta) u_{1}(t)+u_{2}(t)}
\end{aligned}
$$

where $f_{1}$ and $f_{2}$ are two fractional-linear (Möbius) transformations corresponding to $A_{1}$ and $A_{2}$

$$
\begin{aligned}
& f_{1}(\delta)=\frac{a_{1} \delta+b_{1}}{c_{1} \delta+d_{1}} \\
& f_{2}(\delta)=\frac{a_{2} \delta+b_{2}}{c_{2} \delta+d_{2}} .
\end{aligned}
$$

The group $M$ generated by $f_{1}$ and $f_{2}$ is a subgroup of the group of Möbius transformation, and is called the monodromy group of the Riccati equation (1).

Suppose that $\left(\tilde{u}_{1}(t), \tilde{u}_{2}(t)\right)$ is another fundamental solution system of equation (3), and that $\tilde{G}$ is the monodromy group of equation (3) corresponding to this fundamental system, then there exists a nonsingular matrix $T$ such that $\left(\tilde{u}_{1}(t), \tilde{u}_{2}(t)\right)=\left(u_{1}(t), u_{2}(t)\right) T$. So $\tilde{G}=T^{-1} G T$. This means, in view of isomorphism, that the monodromy group $G$ of equation (3) is independent of the choice of the fundamental system of solutions $\left(u_{1}(t), u_{2}(t)\right)$, and so is the monodromy group $M$ of equation (1).

It is easy to check that the monodromy group $M$ of equation (1) and the monodromy group $G$ of the corresponding equation (3) have the same solvability. The structure of a solvable subgroup $G$ of $S L(2, \mathbb{C})$ has been studied in [1]. Here, we state some relevant results as follows.

LEMMA 1. If the monodromy group $G$ of equation (3) is solvable, then its two generators $A_{1}$ and $A_{2}$ must belong to one of the following cases:

$\left(F_{1}\right)$

$$
\left\{\begin{array}{l}
A_{1}=\left(\begin{array}{ll}
a & 0 \\
c & a
\end{array}\right), a^{2}=1, c \neq 0 \\
A_{2}=\left(\begin{array}{ll}
b & 0 \\
d & b
\end{array}\right), b^{2}=1
\end{array}\right.
$$


$\left(F_{2}\right)$

$$
\left\{\begin{array}{l}
A_{1}=\left(\begin{array}{ll}
a & 0 \\
c & a
\end{array}\right), \quad a^{2}=1, \quad c \neq 0 \\
A_{2}=\left(\begin{array}{ll}
b & 0 \\
d & b
\end{array}\right), \quad b^{2} \neq 1
\end{array}\right.
$$

$\left(F_{3}\right)$

$$
\left\{\begin{array}{l}
A_{1}=\left(\begin{array}{cc}
a & 0 \\
0 & a^{-1}
\end{array}\right), a^{2} \neq 1 \\
A_{2}=\left(\begin{array}{cc}
b & 0 \\
0 & b^{-1}
\end{array}\right)
\end{array}\right.
$$

$\left(F_{4}\right)$

$$
\left\{\begin{array}{l}
A_{1}=\left(\begin{array}{cc}
a & 0 \\
0 & a^{-1}
\end{array}\right), a^{2}= \pm i \\
A_{2}=\left(\begin{array}{cc}
0 & b \\
-b^{-1} & 0
\end{array}\right)
\end{array}\right.
$$

$\left(F_{5}\right)$

$$
\begin{cases}A_{1}=\left(\begin{array}{cc}
a & 0 \\
0 & a^{-1}
\end{array}\right), & a^{2}= \pm i \\
A_{2}=\left(\begin{array}{ll}
d & b \\
c & d
\end{array}\right), & b c d \neq 0\end{cases}
$$

$\left(F_{6}\right)$

$$
\left\{\begin{array}{l}
A_{1}=\left(\begin{array}{cc}
a & 0 \\
0 & a^{-1}
\end{array}\right), \quad a^{4} \neq 1 \\
A_{2}=\left(\begin{array}{cc}
0 & b \\
-b^{-1} & 0
\end{array}\right)
\end{array}\right.
$$

$\left(F_{7}\right)$

$$
\begin{cases}A_{1}=\left(\begin{array}{cc}
a & 0 \\
0 & a^{-1}
\end{array}\right), & a^{2}= \pm i \\
A_{2}=\left(\begin{array}{cc}
0 & b \\
-b^{-1} & 0
\end{array}\right) \text { or }\left(\begin{array}{ll}
b & d \\
0 & b
\end{array}\right), & d \neq 0\end{cases}
$$

$\left(F_{8}\right)$

$$
\left\{\begin{array}{l}
A_{1}= \pm E \\
A_{2} \in S L(2, \mathbb{C})
\end{array}\right.
$$

From Lemma 1, it follows that

LEMMA 2. If the monodromy group $M$ of equation (1) is solvable, then its generators must belong to one of the following cases:

$\left(R_{1}\right)$

$$
\left\{\begin{array}{l}
f_{1}=\delta /\left(\sigma_{1} \delta+1\right) \\
f_{2}=\delta /\left(\sigma_{2} \delta+\sigma_{3}\right)
\end{array}, \quad \sigma_{1} \sigma_{3} \neq 0\right.
$$

(corresponding to the cases $\left(F_{1}\right)$ and $\left(F_{2}\right)$ );

$\left(R_{2}\right)$

$$
\begin{cases}f_{1}=\sigma_{1} \delta, & \sigma_{1} \neq 0 \\ f_{2}=\sigma_{2} \delta+\sigma_{3}, & \sigma_{2} \neq 0\end{cases}
$$


(corresponding to the cases $\left(F_{3}\right),\left(F_{7}\right)$ and $\left(F_{8}\right)$ );

$\left(R_{3}\right)$

$$
\begin{cases}f_{1}=\sigma_{1} \delta, & \sigma_{1} \neq 0 \\ f_{2}=\sigma_{2} / \delta, & \sigma_{2} \neq 0\end{cases}
$$

(corresponding to the cases $\left(F_{4}\right)$ and $\left(F_{6}\right)$ );

$\left(R_{4}\right)$

$$
\begin{cases}f_{1}=\sigma_{1} \delta, & \sigma_{1} \neq 0 \\ f_{2}=\delta /\left(\sigma_{2} \delta+1\right), & \sigma_{2} \neq 0\end{cases}
$$

(corresponding to the cases $\left(F_{7}\right)$ and $\left(F_{8}\right)$ ),

$\left(R_{5}\right)$

$$
\left\{\begin{array}{l}
f_{1}=-\delta \\
f_{2}=\left(\delta+\sigma_{1}\right) /\left(\sigma_{2} \delta+1\right), \quad \sigma_{1} \sigma_{2} \neq 0
\end{array}\right.
$$

(corresponding to the case $\left(F_{5}\right)$ ).

LEMMA 3. If the monodromy group $M$ of the Riccati equation (1) is solvable, then there exists an elliptic function solution of the equation.

PROOF. Suppose that $M$ is solvable. From Lemma 2, it follows that

Case 1. If $f_{1}, f_{2}$ belong to $\left(R_{1}\right)$ or $\left(R_{4}\right)$, then $\delta=0$ is the $M$-invariant (i.e. $g(0)=0, \forall g \in M$ ) Therefore, the corresponding solution $z_{2}=-u_{2}^{\prime} / u_{2}$ of equation (1) is single-valued and with double periods $\omega_{1}=2 \alpha, \omega=2 i \alpha$.

It remains to verify that any singularity of $z_{2}(t)$ is a pole. In fact, in the neighborhood of any fixed point, say, $P_{1}: t=t_{1}=-\alpha-i \alpha$, there are two regular solutions of the corresponding Fuchsian equation (3) (see [5])

$$
w_{1}(t)=\left(t-t_{1}\right)^{r_{1}} \phi_{1}(t)
$$

and

$$
w_{2}(t)=\left(t-t_{1}\right)^{r_{2}} \phi_{2}(t)+\theta w_{1}(t) \ln \left(t-t_{1}\right),
$$

where $r_{1}>0, r_{2}<0$ are two real roots of the algebraic equation

$$
r(r-1)-\lambda=0,
$$

$\phi_{1}(t)$ and $\phi_{2}(t)$ are holomorphic in the neighborhood of $t_{1}$, and

$$
\theta=\operatorname{Res}\left(t_{1}, \frac{c}{w_{1}^{2}(t)}\right)
$$

Then in the neighborhood of $t_{1}, u_{2}(t)$, as a solution of equation (3), can be expressed by a linear combination of $w_{1}(t)$ and $w_{2}(t)$. However, as we have indicated above, $z_{2}(t)$ is single-valued, thus the point $P_{1}$ should be a pole of $z_{2}(t)$. The isolated zeros of $u_{2}(t)$ are also poles of $z_{2}(t)$. Besides, there is no other singularity for $z_{2}(t)$. So $z_{2}(t)=-u_{2}^{\prime}(t) / u_{2}(t)$ is an elliptic function solution of equation (1)

Case 2. If $f_{1}$ and $f_{2}$ are of the form $\left(R_{2}\right)$, then $\delta=\infty$ is the $M$-invariant. The same method as in Case 1 for $z_{2}$, works for $z_{1}(t)=-u_{1}^{\prime}(t) / u_{1}(t)$ which is therefore verified to be an elliptic function with periods $2 \alpha$ and $2 i \alpha$.

Case 3. If $f_{1}$ and $f_{2}$ are of the form $\left(R_{3}\right)$

$$
\begin{cases}f_{1}=\sigma_{1} \delta, & \sigma_{1} \neq 0 \\ f_{2}=\sigma_{2} / \delta, & \sigma_{2} \neq 0,\end{cases}
$$

then it is easy to verify that for any $\delta \in \widehat{\mathbb{C}}$, 


$$
\begin{gathered}
f_{2}^{2}(\delta)=f_{2}\left(f_{2}(\delta)\right)=\delta, \\
f_{2}^{-1} f_{1}^{-1} f_{2} f_{1}(\delta)=\delta,
\end{gathered}
$$

where $f_{2}^{-1}$ denotes the inverse transformation of $f_{2}$. This means that $z_{1}=-u_{1}^{\prime} / u_{1}$ and $z_{2}=-u_{2}^{\prime} / u_{2}$ are both single-valued solutions with periods $2 \alpha$ and $4 i \alpha$. Further, with the same verification as in Case 1, we get that $z_{1}(t)$ and $z_{2}(t)$ are both elliptic functions.

Case 4. If $f_{1}$ and $f_{2}$ are of the form $\left(R_{5}\right)$

$$
\left\{\begin{array}{l}
f_{1}=-\delta \\
f_{2}=\left(\delta+\sigma_{1}\right) /\left(\sigma_{2} \delta+1\right), \quad \sigma_{1} \sigma_{2} \neq 0
\end{array}\right.
$$

then it is easy to check that for any $\delta \in \widehat{\mathbb{C}}$

$$
\begin{gathered}
f_{1}^{2}(\delta)=\delta \\
f_{2}^{-1} f_{1}^{-1} f_{2} f_{1}(\delta)=\delta
\end{gathered}
$$

and that

$$
f_{2}\left(\delta_{2}\right)=\delta_{2}, \quad i=1,2,
$$

where $\delta_{1}=\sqrt{\frac{\sigma_{1}}{\sigma_{2}}}, \delta_{2}=-\delta_{1}$ This means that

$$
z_{+}=-\frac{\delta_{1} u_{1}^{\prime}+u_{2}^{\prime}}{\delta_{1} u_{1}+u_{2}} \text { and } z_{-}=-\frac{\delta_{2} u_{1}^{\prime}+u_{2}^{\prime}}{\delta_{2} u_{1}+u_{2}}
$$

are both elliptic function solutions with periods $4 \alpha$ and $2 i \alpha$. This completes the proof.

We note the following facts:

(a) If the Riccati equation (1) has an elliptic function solution, say, $z_{1}(t)$, then its general solution can be expressed as

$$
z(t)=z_{1}(t)+y(t)
$$

where $y(t)$ satisfies the following Bernoulli's equation.

$$
y^{\prime}(t)=y^{2}(t)+2 z_{1}(t) y(t) .
$$

(b) Any elliptic function with double periods $\omega_{1}$ and $\omega_{2}$ is of the form

$$
R_{1}[\wp(t)]+R_{2}[\wp(t)] \wp^{\prime}(t)
$$

where $\wp(t)$ is the Weierstrass elliptic function with the same double periods, $\wp^{\prime}(t)$ is its derivative, and $R_{1}(x), R_{2}(x)$ are rational functions in $x$ with constant coefficients (see [11]).

By Lemma 3 and the facts above, we obtain immediately:

THEOREM. If the monodromy group of the Riccati equation (1) is solvable, then equation (1) is integrable, that is to say, its general solution can be expressed in terms of a Weierstrass elliptic function by solving algebraic equations, differentiation and integration in finite terms; each solution of equation (1) is meromorphic on $\mathbb{C}$.

By remarking the following facts: (i) for $\lambda=n(n-1)(n \in \mathbb{N})$, the monodromy group $G$ of equation (3) is solvable (see [1,9]), (ii) the solvability of the group $M$ is equivalent to that of the group $G$, we have also:

COROLLARY 1. For $\lambda=n(n-1)(n \in \mathbb{N})$, the Riccati equation (1) is integrable (Recent work has shown that the Riccati equation (1) is also integrable when $\lambda=\frac{(4 n-1)(4 n-3)}{4}, n \in \mathbb{N}$. This new result was presented by Guan at Mathematics Today and Tomorrow International Conference in Florida in 1997). 
Especially, we have:

COROLLARY 2. For $\lambda=6$, the Riccati equation (1) is integrable. Its general solution can be expressed in terms of the Weierstrass elliptic function of order 3 with periods $2 \alpha$ and $2 i \alpha$

PROOF. Suppose that $\tilde{z}(t)$ is the solution of the initial value problem

$$
\left\{\begin{aligned}
z^{\prime} & =z^{2}-6 \wp(t) \\
z(0) & =0
\end{aligned}\right.
$$

Under the transform $z=-u^{\prime} / u$, this solution $\tilde{z}(t)$ corresponds to the solution $\tilde{u}(t)$ of the following problem

$$
\left\{\begin{array}{l}
u^{\prime \prime}-6 \wp(t) u=0 \\
u(0)=0,
\end{array} u^{\prime}(0)=0 .\right.
$$

It has been shown (see [9]) that $\tilde{u}(t)$ has the following properties (i) $\tilde{u}(t+2 \alpha)=-\tilde{u}(t)$, and $\tilde{u}(t+2 i \alpha)=-\tilde{u}(t) ;$ (ii) the points $t_{m, n}=(2 m-1) \alpha+(2 n-1) i \alpha(m, n \in \mathbb{N})$ are all poles of order 2 for $\tilde{u}(t)$; (iii) the points $\tilde{t}_{m, n}=(2 m-1) \alpha+2 n i \alpha$ and $\widehat{t}_{m, n}=2 m \alpha+(2 n-1) i \alpha$ are the simple zeros of $\tilde{u}(t)$, and that $\tilde{u}(t)$ does not have any other singularity or zero on $\mathbb{C}$. These properties imply that $\tilde{z}(t)=-\tilde{u}^{\prime}(t) / \tilde{u}(t)$ is an elliptic function of order 3 with periods $2 \alpha$ and $2 i \alpha$. This completes the proof

The discussion of the particular doubly periodic Riccati equation (1) can be extended to a more general case, that is, for any Riccati equation with elliptic functions as its coefficients, its integrability defined by the solvability of its monodromy group means that its general solution can be expressed in terms of some relevant elliptic function by solving algebraic equations, differentiation and integration in finite terms. However, the remaining problem, that is, how to construct its monodromy group directly from its coefficients, is still open. (In the particular case, i.e. for equation (1), the exact relation between the monodromy group and the parameter $\lambda$ has been obtained. This result was presented by Guan at Mathematics Today and Tomorrow International Conference of Mathematics in Florida in 1997 )

\section{REFERENCES}

[1] KE-YING, G. and SHAO-FEI, Z., The structure of solvable subgroup of $S L(2, \mathrm{C})$ and integrability of a Fuchsian equation on Torus $T^{2}$, Science in China, 39 (5) (1996), 501-508.

[2] WATSON, G.N., A Treatise on the Theory of Bessel Function, 2nd ed., Cambridge, 1944, 11 123.

[3] OLVER, P.J , Applications of Lie Groups to Differential Equations, Springer-Verlag, New York, 1986.

[4] KOLCHIN, E.R., Differential Algebra and Algebraic Groups, Academić Press, 1973.

[5] GOLUBEC, V.V., Lectures on analytic theory of differential equations, Gos. Izd. Tekh. Teor. Lit., Moscow-Leningrad (1950) (Russian).

[6] ANOSOV, D.V. and ARNOLD, V.I., Dynamical Systems I, Springer-Verlag, Berlin-Heidelberg, 1988.

[7] KE-YING, G., Some developments in the qualitative investigation to ordinary differential equations in the complex domain, Proceedings on the Theory and Applications of Ordinary Differential Equations, Science Press, Beijing (1992), 46-52 (in Chinese).

[8] KE-YING, G. and RU-YI, CHENG, Global first integration and admitted Lie group of second order polynomial system in the complex domain, Journal of Nanjing University, Mathematical Biquarterly (1993), 229-235.

[9] LING, M., ZHAOXIA, Y. and KE-YING, G., Integrability of second-order Fuchsian equation on Torus $T^{2}$, Chinese Science Bulletin, 41 (7) (1996), 534-538.

[10] HILLE, E., Analytic Function Theory, Ginn and Company, Boston, 1962 


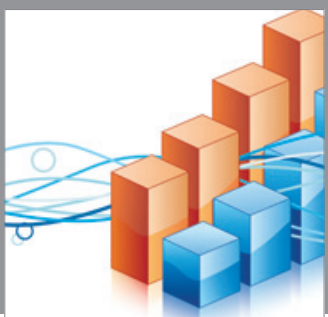

Advances in

Operations Research

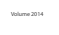

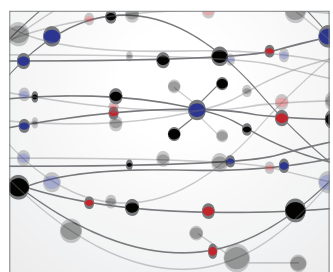

\section{The Scientific} World Journal
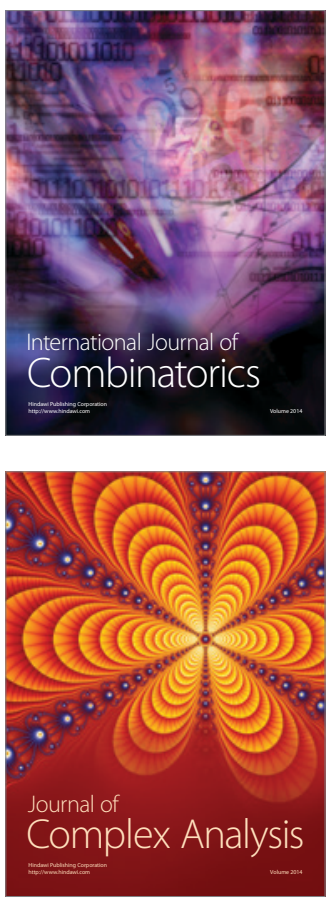

International Journal of

Mathematics and

Mathematical

Sciences
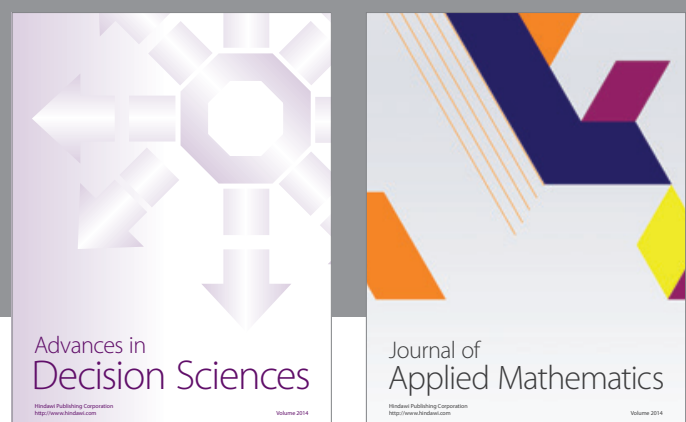

Journal of

Applied Mathematics
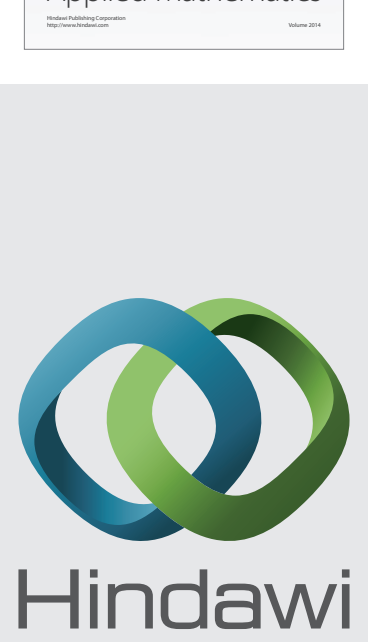

Submit your manuscripts at http://www.hindawi.com
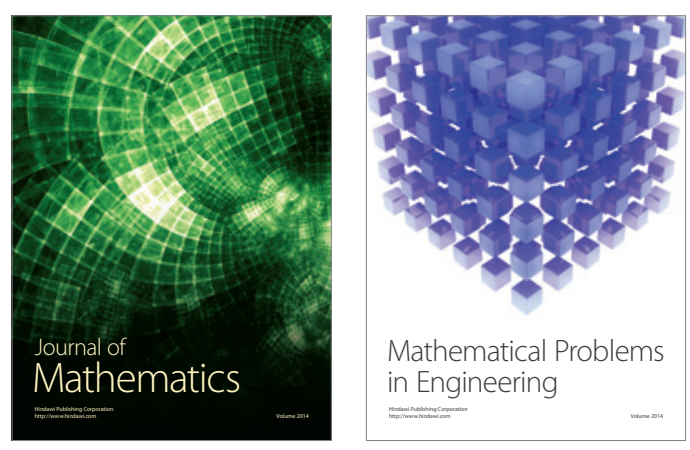

Mathematical Problems in Engineering
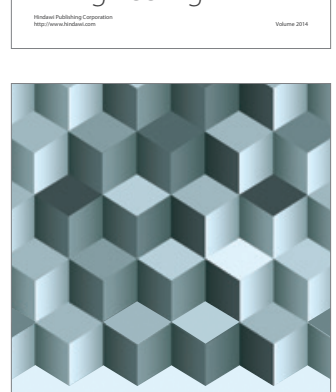

Journal of

Function Spaces
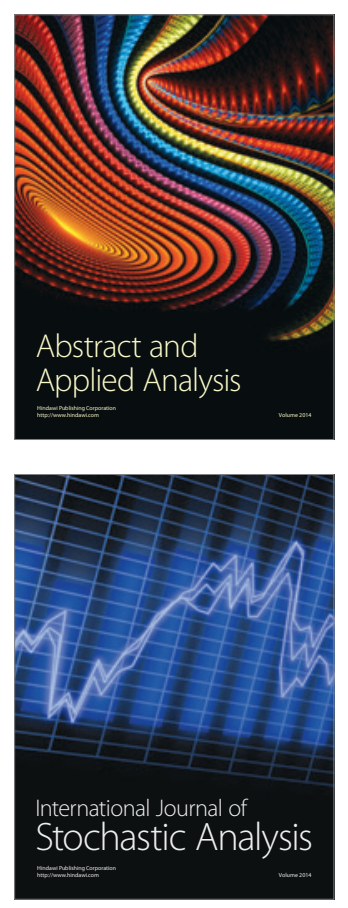

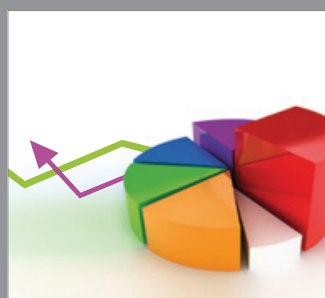

ournal of

Probability and Statistics

Promensencen
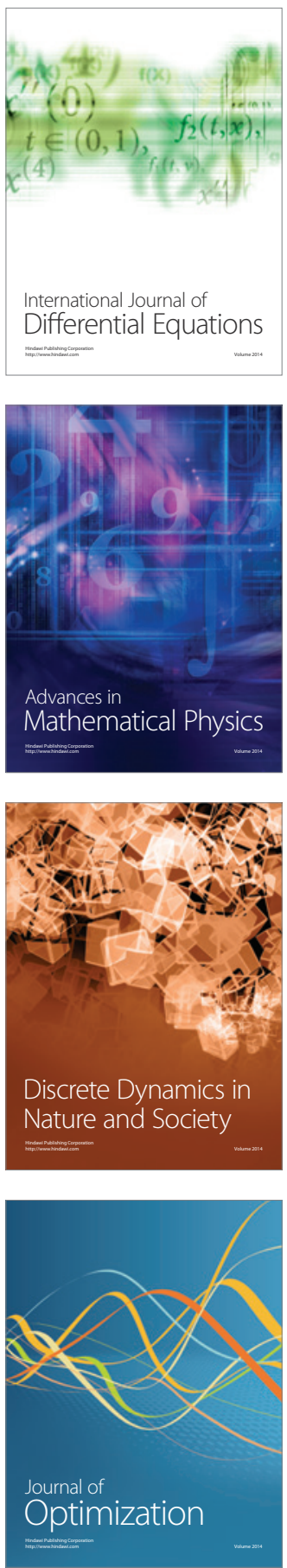